\title{
A Brief Report of Polymicrobial Osteomyelitis of Odontoid Process with Epidural Abscess
}

\author{
Willem Guillermo Calderon-Miranda ${ }^{1}$, Nidia Escobar Hernandez ${ }^{2}$, Luis Rafael Moscote-Salazar ${ }^{3}$ * \\ ${ }^{1}$ Resident of Radiology, Universidad Nacional Autonoma de Mexico, Mexico D.F, Mexico \\ ${ }^{2}$ Chief of Radiology Department, Hospital General Manuel GEA Gonzalez, Mexico D.F, Mexico \\ ${ }^{3}$ Neurosurgeon, Red Latino. Latin American Trauma \& Intensive Neuro-Care Organization. Bogota, Colombia \\ * Corresponding Author Address: Department of Neurosurgery, Red Latino, Latin American Trauma and Intensive Neuro-Care Organization, \\ Bogota, Colombia. E-mail: mineurocirujano@aol.com
}

Article Type: Brief Report

Received: December 13, 2015, Last revised: December 15, 2015, Accepted: December 25, 2015

\section{Abstract}

Background \& Importance: Infections of the craniocervical junction are rare.

Case Presentation: We present a case of infection by methicillin-sensitive Staphylococcus aureus and Streptococcus mitis that was not previously reported.

Conclusion: Neurosurgeons must suspect for diagnosis and initiate broad antimicrobial therapy, including active agents against gram-negative and then initiate a targeted therapy. The purpose of this report is to highlight the importance of early diagnosis for a successful medical treatment.

Keywords: Osteomyelitis; Spinal cord compression; Epidural abscess; Odontoid process; Cervical spine abscess

Please cite this paper as: Calderon-Miranda W.G, Hernandez N.E, Moscote-Salazar L.R. A Brief Report of Polymicrobial Osteomyelitis of Odontoid Process with Epidural Abscess. Iran. J. Neurosurg. 2015;1(3):33-34.

\section{Introduction}

Osteomyelitis of the odontoid process associated with epidural abscess is a rare clinical entity characterized by the presence of inflammatory and infectious paranasal or near process with intracranial extension. The existence of predisposing factors for becoming immunocompromised such as diabetes mellitus, HIV infection and steroid use are common.

\section{Case Presentation}

A 55-year-old male patient consuming hallucinogenic drugs (cocaine, marijuana) by inhalation, who developed pain and neck stiffness was studied. He was treated with anti-inflammatory and analgesic for 25 days, but for worsening symptoms he had been refereed to our institution, presenting hallucinations and dysarthria. A physical assessment was central glucose of $851 \mathrm{mg} / \mathrm{dl}$ and deterioration of neurological status, which required mechanical ventilatory support. MRI of the junction craniovertebral showed a hyperdense epidural mass and presence of air and left odontoid subluxation with Grisel syndrome secondary to inflammation in the parapharyngeal area. Chest radiography showed left pneumothorax of approximately $80 \%$, which was successfully managed with chest tube. MRI with gadolinium showed the craniovertebral epidural abscess around the odontoid process without compression and parafacetario cervicomedullary abscess on the left side of verterbral bodies C2-C3 (Figure 1 A, B, C).
In the cerebrospinal fluid culture a methicillin-sensitive Staphylococcus aureus and Streptococcus mitis were isolated, treated with ceftriaxone, and metronidazole-dicloxacillin. On the seventh day of stay in critical care unit, successful extubation was achieved. He recovered from mild neck stiffness after completing antibiotic therapy.

\section{Discussion}

Osteomyelitis of the odontoid process is very rare and therefore requires a high degree of clinical suspicion for diagnosis (2-4). The diagnosis requires a careful assessment of history, comorbid conditions, laboratory tests and imaging. Late diagnosis can lead to poor prognosis. MRI is the first imaging modality. The occipitocervical immobilization, broad-spectrum antibiotic therapy and surgical stabilization in selected cases are the foundations of treatment $(5,6)$.

\section{Conclusion}

Osteomyelitis of the odontoid process is an entity of difficult diagnosis that may prove catastrophic when accompanied by epidural abscess. Our patient had a satisfactory condition, demonstrating the beneficial effect of directed antibiotic treatment. 

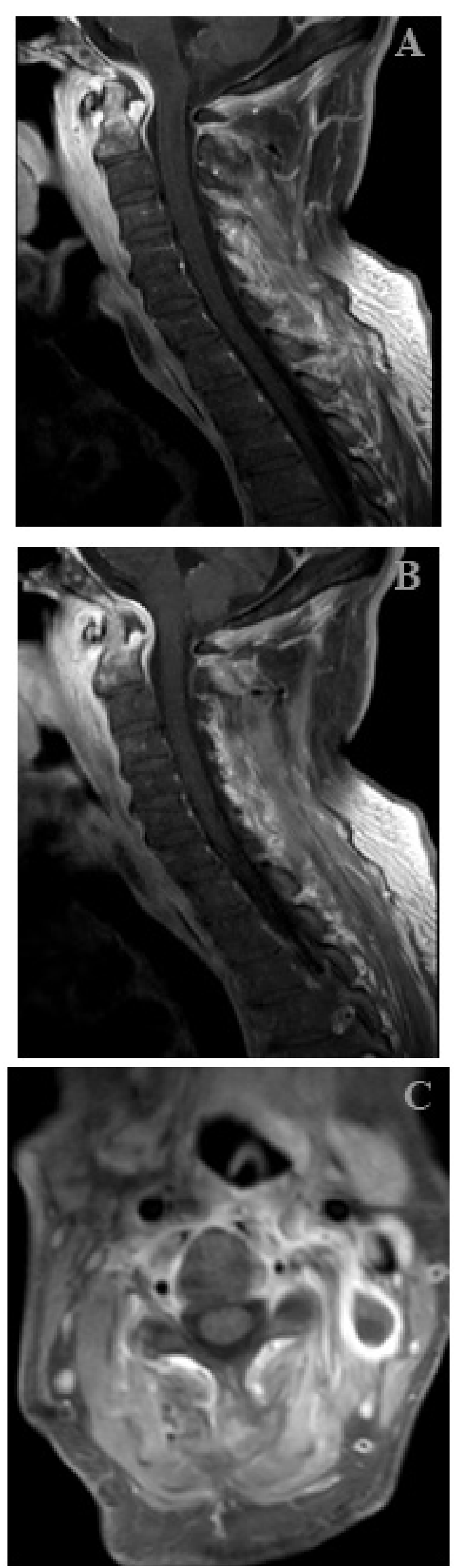

Figure 1. T2-weighted MRI image showing a hyperintense epidural abscess and showing hypointensity of the odontoid process

\section{References}

1. Kaufmann DM, Kaplan JG, Litman N Infectious agents in spinal epidural abscesses . Neurology 1980. 30: 844-850

2.Haridas A, Walsh DC, Mowle DH. Polymicrobial Osteomyelitis of the Odontoid Process with Epidural Abscess: Case Report and Review of Literature. Skull Base. 2003 May;13(2):107-111.

3. Kubo S, Takimoto H, Hosoi K, Toyota S, Karasawa J, Yoshimine T. Osteomyelitis of the odontoid process associated with meningitis and retropharyngeal abscess--case report. Neurol Med Chir (Tokyo). 2002 Oct;42(10):447-51.

4. Wiedau-Pazos M, Curio G, Grüsser C. Epidural abscess of the cervical spine with osteomyelitis of the odontoid process. Spine (Phila Pa 1976). 1999 Jan 15;24(2):133.

5. Willenborg KM, Stöver T, Becker H, Krauss JK, Lenarz T. Parapharyngeal abscess and osteomyelitic destruction of the odontoid process. Laryngorhinootologie. 2007 Feb;86(2):128-30.

6. Young WF, Weaver M. Isolated pyogenic osteomyelitis of the odontoid process. Scand J Infect Dis. 1999;31(5):512-5. Review. 